\title{
White Matter Changes in Cerebrovascular Disease: Leukoaraiosis
}

\author{
Anca Hâncu, Irene Răşanu and Gabriela Butoi \\ County Emergency Clinical Hospital Constanta, \\ Romania
}

\section{Introduction}

The term 'leukoaraiosis', derived from the Greek 'leuko' meaning white and 'araios' meaning rarefied, was introduced in 1987, as a "neutral term, exact enough to define whitematter changes in the elderly or the demented, general enough that it serves as a description and a label, and demanding enough that it calls for a precise clinical and imaging description accompanied when possible by pathologic correlations" (Hachinski et al., 1987).

When the term leukoaraiosis (LA) was introduced, only CT imaging was widely available. Similar appearance is conspicuous, and more florid on T2-weighted magnetic resonance imaging (MRI), particularly on fluid attenuated inversion recovery (FLAIR) images. Leukoaraiosis is currently defined as diffuse, confluent white matter abnormality (low density on CT, hyperintensity on T2-weighted or FLAIR MRI), often with irregular margins, commonly seen in the normal elderly and in association with vascular risk factors such as hypertension, or in context of cognitive impairment. The term was introduced to avoid confusing an imaging appearance with a specific pathology ( $\mathrm{O}^{\prime}$ Sullivan, 2008). Leukoaraiosis can be focal, patchy or diffuse area in the white matter and it is located periventricularly or deeper in the white matter.

Leukoaraiosis severity has traditionally been graded by visual scales. Simples scales like that of van Swieten divide the appearances into only two grades of severity; more complex scales like the Fazekas scale discriminates "punctate", "early confluent" and "confluent" white matter lesions, while the Sheltens scale adopts a 0-6 scale in multiple anatomical regions (including periventricular and nonperiventricular white matter lesions - WML; periventricular hyperintensities are further separated into frontal, occipital, and lateral aspects) (O' Sullivan, 2008; Scheltens et al., 1993, as cited by Bohnen et al., 2009). Other rating scales of WML are the Brant-Zawadzki Scale and the Cardiovascular Health Study Scale both of which place relatively more emphasis on periventricular WML (Bohnen et al., 2009). However, even fully quantitative volumetric measurements of leukoaraiosis correlate weakly with cognitive and physical function, suggesting that T2-weighted MRI provides only a rough impression of the severity of the underlying pathology ( $\mathrm{O}^{\prime}$ Sullivan, 2008).

T2-weighted imaging is sensitive to liquid, gliosis and the effects of demyelination. FLAIR images are heavily T2-weigtened with cerebrospinal fluid suppression. This makes it 
possible to detect also leukoaraiotic lesions situated close to the cerebrospinal fluid and to differentiate Virchow-Robin spaces from leukoaraiosis. The decline in cerebral blood flow in areas with leukoaraiosis can be detected with imaging techniques such as average apparent diffusion coefficient (ADCav) where tissues with faster diffusion appear bright and tissues with slower diffusion dark. Normally axons produce significant hindrance to water diffusion but leukoaraiosis causes axonal loss and furthermore leads to an increase in water content of the tissue which can be detected with these imaging techniques. DWI (diffusion weighted MRI) makes it possible to differentiate acute and chronic ischemic stroke lesions from leukoaraiosis. In DW images, tissues with faster diffusion appear dark and tissues with slower diffusion bright (Helenius et al., 2002, as cited by Kurkinen, 2009).
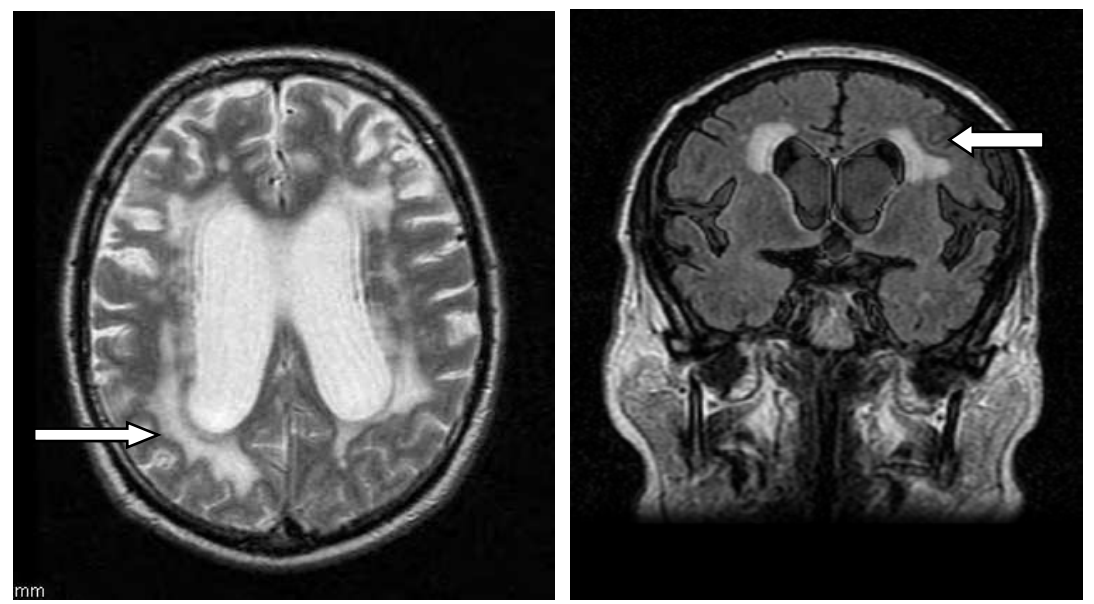

Fig. 1. Cerebral MRI scan showing leukoaraiosis (arrows); left: axial T2 weighted; right: coronal FLAIR sequence.
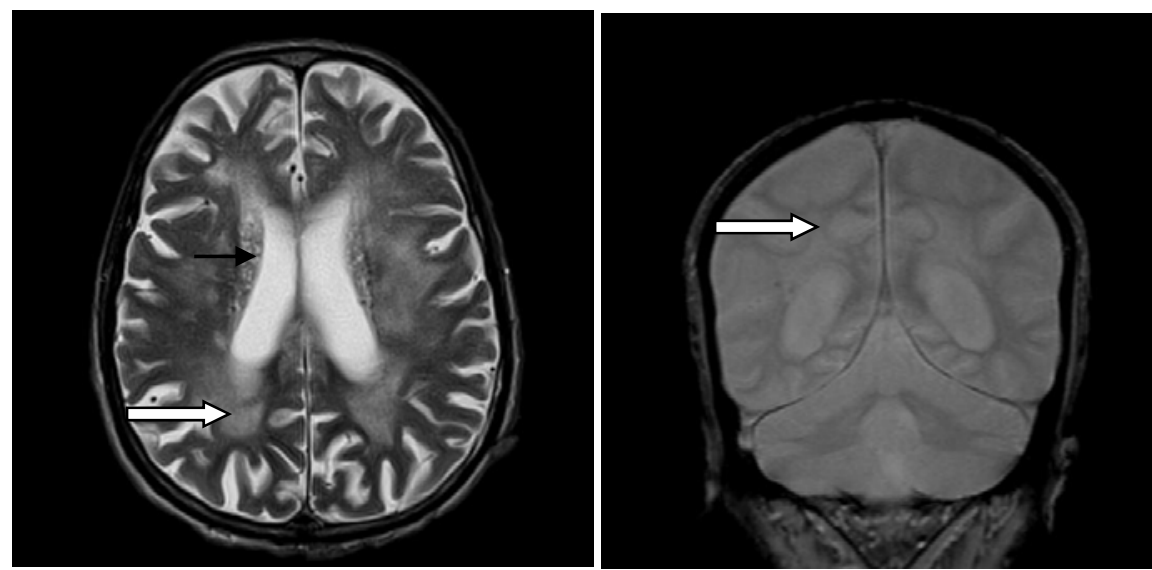

Fig. 2. MR images from a 76-year old patient; left: T2- weighted sequence showing leukoaraiosis (white arrow) and perivascular dilated spaces (black arrow); right: Proton density sequence showing leukoaraiosis (white arrow). 
Newer MRI sequences can provide useful additional information. Diffusion-weighted imaging, for example, allows the distinction of new lacunar infarcts from background leukoaraiosis. In patients who presents with intracerebral haemorrhage, especially lobar haemorrhage, gradient acho (T2*-weighted) images should be performed to look for evidence of previous haemorrhages or microbleeds. In terms of quantifying white matter damage, several techniques (such as diffusion tensor MRI) are proving superior to T2weighting imaging. Diffusion tensor MRI provides a much better index of white matter damage, and simple whole-brain measurements, such as diffusion histograms, can help track disease progression. Diffusion tensor MRI also demonstrates the variability in the extent of white matter disruption both within lesions and in normal-appearing white matter (O’ Sullivan, 2008).

\section{Epidemiology of leukoaraiosis}

Several studies have described the prevalence of leukoaraiosis in different population groups, but there is considerable variability in the published figures. This variability can be attributed to the heterogeneity of age and vascular risk factors of patients with different imaging modalities used and differences in the scales used to define the leukoaraiosis. Often, when first discovered, white matter hyperintensities occur in the context of relatively normal brain function. But these lesions are not normal as they indicate an increased risk for stroke, cognitive decline, dementia, and death, as reported by Debette and Markus (2010) at the end of their metanalysis of the existing literature through November 2009. They examined 46 prospective longitudinal studies and found that white matter hyperintensities predict an increased risk of stroke (hazard ratio 3.3), dementia (hazard ratio 1.9) and death 2.0 (hazard ratio 2.0).

Roppele et al. (2010) performed magnetization transfer imaging in 328 neurologically asymptomatic Austrian Stroke Prevention Study participants (age range, 52-87 years). FLAIR was used to delineate white matter hyperintensities and to define normal-appearing brain tissue. The magnetization transfer ratio was measured globally in normal-appearing brain tissue by using a histogram analysis technique and focally in white matter hyperintensities. Associations of magnetization transfer ratio metrics with sex and a large battery of different cerebrovascular risk factors (age, arterial hypertension, diabetes mellitus, smoking, body mass index, cholesterol and triglyceride levels, glycated hemoglobin, and the presence of cardiac disease) were assessed with univariate and multiple regression analysis.

Age was seen to affect all magnetization transfer ratio histogram metrics of normalappearing brain tissue, and a faster decrease of the magnetization transfer ratio peak height occurred in men. Independent associations with magnetization transfer ratio metrics were found for arterial hypertension and diabetes mellitus. Besides lesion grade, arterial hypertension was also significantly associated with a lower magnetization transfer ratio in white matter hyperintensities.

\section{Pathophysiology of leukoaraiosis}

The pathogenesis of leukoaraiosis remains controversial. It is unclear whether the mechanisms are the same for large and small punctate foci and for extensive diffuse 
leukoaraiosis. Moreover, pathological changes could be consequences of reaching the white matter rather than causes of it.

It has been assumed that the ischemic insult, responsible for LA, results from the vulnerable nature of the long penetrating end-arteries that feed the deep white matter. The deep brain structures (white matter and deep gray nuclei) are supplied by perforating arteries that are end-arteries with no collateral supply. These penetrating arteries do not arborise but give off perpendicularly oriented short branches that irrigate the white matter, each of which provides the blood supply to a cylindrically shaped metabolic unit. In the region between the cortical and ventricular surfaces, centripetal and centrifugal penetrating arteries from an internal watershed area lacking anastomoses is particularly susceptible to being injured as a result of systemic or focal decreases in cerebral blood flow (Rowbotham et al., 1965, De Reuck, 1971, as cited by Birns \& Kalra, 2008).

At the vascular level, the continuum extends from the findings of hyaline thickening of the walls of small arteries to the lipohyalinosis (a major disruption in wall with infiltration of macrophages and luminal narrowing). At the level of the parenchyma, there is a loss of myelin and axons with a glial reaction and even areas of infarction (Fazekas et al., 1993, Lotz et al., 1986).

The main pathogenic hypotheses involve:

\subsection{Ischemia, cerebral vasoreactivity and autoregulation}

The pathological studies suggest that leukoaraiosis is one manifestation of cerebral small vessel disease. This is supported by strong pathological and clinical associations with the other major manifestation of small vessel disease-lacunar stroke. The pathogenesis of cerebral small vessels disease is still a matter of investigation but both clinical and pathological studies support the most popular hypothesis that acute disruption of blood supply in one arterial territory results in lacunar infarction while a more chronic and widespread reduction in perfusion causes leukoaraiosis. This is consistent with the spatial pattern and distribution of leukoaraiosis, which arises first in those areas furthest from the origin of the arterioles in the periventricular and deep white matter regions (Hassan et al., 2003, Pantoni, 2002, as cited by Birns \& Kalra, 2008).

Reductions in white matter perfusion in leukoaraiosis have been demonstrated using xenonCT, MRI, PET and SPECT and in some studies, the degree of hypoperfusion has been found to correlate with the severity of leukoaraiosis (Oishi et al., 1999, Miyazawa et al., 1997, as cited by Birns \& Kalra, 2008 ). Furthermore, quantitative perfusion and diffusion tensor MRI studies have revealed reduced cerebral blood flow in normal appearing white matter in periventricular regions in patients with leukoaraiosis, suggesting that areas are "at risk." $\left(\mathrm{O}^{\prime}\right.$ Sullivan, 2001, 2002). Two studies on the relationship between cerebrovascular reactivity and cerebral small vessels disease (which excluded patients with carotid artery stenoses or those undertoken soon after an acute ischaemic event, knowing the effect of these comorbidities on cerebrovascular reactivity) demonstrated impaired reactivity in patients with multiple lacunar infarctions but no association between cerebrovascular reactivity and the severity of leukoaraiosis on brain MRI (Molina et al., 1999, Cupini et al., 2001 as cited by Birns \& Kalra, 2008). 
Supporting the role of ischemia in the pathogenesis of leukoaraiosis, the results of a study showed a statistically significant correlation between the presence and severity of leukoaraiosis and degree of carotid stenosis. A trend toward increased risk of development of leukoaraiosis in carotids with fatty plaques also was observed. The data confirmed that the development of leukoaraiosis is strongly correlated with age (Saba et al., 2009). A further study showed a statistically significant correlation between increased carotid artery wall thickness and LA (and its severity) (Saba et al., 2011).

\subsection{Endothelial dysfunction/blood-brain barrier abnormalities}

Histopathological evidence of endothelial cells activation and retraction with increased vascular permeability, increased circulating levels of leukocyte adhesion molecules such as ICAM1 (intercellular adhesion molecule-1) and E selectin (shed from the surface of activated endothelial cells) and a rise in the markers of coagulation activation (including thrombinantithrombin complex and prothrombin fragments $1+2$ ) have been reported in patients with small vessels desease compared with controls (Lin et al., 2000, Hassan et al., 2003, Fassbender et al., 1999, Tomimoto et al., 1999, as cited by Birns \& Kalra, 2008). In addition, serum concentration of thrombomodulin and von Willebrand factor, which are both molecular markers of endothelial cell damage, have been shown to correlate with MRI evidence of small vessels disease (Ishii et al., 1991, Kohriyama et al., 1996 as cited by Birns \& Kalra, 2008).

A number of studies have shown that chronic hypertension predisposed to impaired bloodbrain barrier function, with endothelial cell retraction, increased vascular permeability and greater susceptibility to white matter injury for relatively small insults (Tomimoto et al., 1996, Lin et al., 2000, Pantoni, 2002, Wardlaw et al., 2003, Birns et al., 2005, as cited by Birns \& Kalra, 2008).

In patients with subcortical white matter disease, plasma proteins have been found in the tissue around perforating arteries and increased concentrations of a number of proteins in the cerebro-spinal fluid have been found compared with controls, supporting the idea that the proteins reached the cerebro-spinal fluid via leakage from small perforating arteries (Akiguchi et al., 1998., Pantoni et al., 1993, as cited by Birns \& Kalra, 2008).

One study has demonstrated intravenously injected contrast agent to leak into the brain, particularly in the territory of the perforating arteries, more in those with leukoaraiosis than in controls (Starr et al., 2003, as cited by Birns \& Kalra, 2008).

\subsection{Disturbances in cerebro-spinal fluid circulation}

Murata et al (1981) hypothesized that disturbances in cerebro-spinal fluid circulation may play a role in the pathogenesis of leukoaraiosis. Roman suggested that increased accumulation of cerebro-spinal fluid in the ventricules raises the interstitial pressure in the periventricular parenchyma, thus causing ischaemia to the white matter (Roman, 1991, Kimura et al., 1992, as cited by Birns \& Kalra, 2008 ).

\subsection{Plasma viscosity}

Schneider et al (1997) showed plasma viscosity to be elevated in patients with leukoaraiosis and lacunar infarction and considered that it may alter cerebro-spinal fluid properties and favour chronic ischaemic white matter damage. 


\subsection{Platelet hyperaggregability and other coagulation abnormalities}

Fujita et al (2011) demonstrated a significantly increased incidence of platelet hyperaggregability in 73 patients with leukoaraiosis compared with 102 controls. Twentyone patients with leukoaraiosis and uncorrected platelet hyper-aggregability were compared with 21 controls matched for age, grade of leukoaraiosis and observation period whose platelet hyper-aggregability was corrected. The results of their study showed that the progress of leukoaraiosis is significantly inhibited by long-term correction of platelet hyperaggregability, suggesting platelet hyper-aggregability as a risk factor for leukoaraiosis

Martí-Fàbregas et al. (2002) investigated whether there is a direct correlation between plasma fibrinogen levels and the amount of leukoaraiosis in 28 patients with symptomatic small-vessel disease. They found a significant correlation between plasma fibrinogen levels and the amount of leukoaraiosis in patients with symptomatic cerebral small-vessel disease. This result suggests that fibrinogen may be involved in the pathophysiology of leukoaraiosis in these patients.

Hassan et al showed that while tissue factor and the ratio of tissue factor to TFPI (tissue factor pathway inhibitor) did not differ significantly between patients with small vessel disease and controls, the tissue factor/TFPI ratio was higher in small vessel disease patients with leukoaraiosis compared with isolated lacunar infarction (Hassan et al., 2003, as cited by Birns \& Kalra, 2008).

\subsection{Cerebral venous circulation impairment}

Some authors found an age-related gradual increase in the thickness of the walls of veins and venules near the lateral ventricles and a striking degree of vessels wall thickening, resulting in narrowed lumina and even occlusion, in patients with leukoaraiosis. The thickened vascular walls stained strongly for collagens I and III. In the studied cases, the degree of venous collagenosis statistically correlated with the severity of leukoaraiosis. The authors questioned whether increased resistance to venous blood flow resulting from the venous stenosis, might induce chronic ischaemia and/or oedema in the deep white matter, perhaps somehow leading to leukoaraiosis, or indeed whether the collagenosis itself occurs as a result of ischaemia (Moody et al., 1995, Brown et al., 2002, as cited by Birns \& Kalra, 2008).

More recently, Chung \& $\mathrm{Hu}$ (2010) hypothesed that chronic cerebral hypoperfusion associated with vasogenic edema, microbleeding or/and endothelial dysfunction found in leukoaraiosis favors venous ischemia, in stead of arterial ischemia, as its pathogenesis. Given that the involved regions in leukoaraiosis (periventricular and subcortical regions) are the drainage territory of deep cerebral venous system and the watershed region between the superficial and deep cerebral venous system respectively, and adding the facts that periventricular venule collagenosis, and retinal and intraparenchymal venules dilatation are related to the severity of leukoaraiosis, the authors suggested that cerebral venous hypertension caused by downstream venous outflow impairment might play a major role in the pathogenesis of leukoaraiosis. Jugular venous reflux is therefore suggested to play a key role in the pathogenesis of leukoaraiosis through a sustained or long-term repetitive retrograde-transmitted cerebral venous pressure and venous outflow insufficiency, which might lead to chronic cerebral venous hypertensions, abnormal cerebral venules structural 
changes, decreased cerebral blood flow, endothelial dysfunction, and vasogenic edema in cerebral white matters.

\subsection{Others hypothesis}

It has been also demonstrated homocysteine (which is toxic to the endothelium) to be a strong risk factor for small-vessel disease on 90 patients with leukoaraiosis and lacunar infarction compared to 52 patients with isolated lacunar infarction after controlling for both conventional risk factors and age (Hassan at al., 2004, Khan et al., 2007, as cited by Birns \& Kalra, 2008).

Matrix metalloproteinases are neutral proteases that disrupt the blood-brain barrier and degrade myelin basic protein under conditions of neuroinflammation. Candelario-Jalil et al., studied 60 patients with suspected vascular cognitive impairment due to small vessel disease (twenty-five of which were classified as subcortical ischemic vascular disease, whereas other groups included mixed Alzheimer disease and vascular cognitive impairment, multiple strokes, and leukoaraiosis when white matter lesions were present and the diagnosis of vascular cognitive impairment was uncertain) by measuring metalloproteinase-2, metalloproteinase-3 and metalloproteinase- 9 activity as well as the albumin level in the cerebrospinal fluid. They found that increased levels of metalloproteinases are associated with increased cerebrospinal fluid albumin and suggest that they may contribute to the pathophysiology of subcortical ischemic vascular disease.

Since necrosis is not obvious in LA lesions, Brown et al. investigated the occurrence of apoptosis. They obtained 1.5-cm-thick coronal brain slices at autopsy from two patients with LA. MRI was performed on the brain slices. Sections were stained by several methods including the terminal deoxynucleotidyl transferase dUTP (uridine 5'-triphosphate) nick end labeling (TUNEL) method for DNA fragmentation. The presence of numerous scattered cells in the LA lesions showing DNA fragmentation suggests that those cells are damaged and dying, at least some by apoptosis. The apoptosis in the white matter adjacent to the LA lesions suggests progressive cell loss and expansion of the LA lesions (Brown et al., 2002).

\subsection{Genetic factors}

RNA expression was assessed in the blood of individuals with and without extensive white matter hyperintensities (WMH) to search for evidence of oxidative stress, inflammation, and other abnormalities described in WMH lesions in brain. Cluster and principal components analyses showed that the expression profiles for almost 300 genes distinguished $\mathrm{WMH}+$ from $\mathrm{WMH}-$ subjects. Function analyses suggested that $\mathrm{WMH}-$ specific genes were associated with oxidative stress, inflammation, detoxification, and hormone signaling, and included genes associated with oligodendrocyte proliferation, axon repair, long-term potentiation, and neurotransmission (Xu et al., 2010).

Fernandez-Cadenas et al. analyzed 212 single nucleotide polymorphisms (SNPs) in 142 patients with ischaemic stroke, generating a total of 30104 genotypes. Seventy-nine subjects (55.6\%) presented leukoaraiosis measured by the Fazekas scale and $69(48.6 \%)$ by agerelated white matter changes (ARWMC) scale. This study revealed that the genes associated with leukoaraiosis were involved in blood-brain barrier (BBB) homeostasis (FernandezCadenas et al., 2010). 


\section{Specific features of LA in the main types of cerebrovascular diseases}

\subsection{Stroke}

Although patients with cerebrovascular disease may have white matter abnormalities related to large-vessel, embolic or ischemic-hypoxic etiologies, by far small-vessel disease is believed to be the most common substrate in case of diffuse, bilateral, preferential white matter involvement (Gomes \& Caplan, 2008).

Leukoaraiosis (LA) is a common finding in stroke (particularly ischemic) and shares similar risk factors and pathophysiologic mechanisms with both ischemic and hemorrhagic stroke. LA may also be an independent predictor of stroke outcomes.

After an acute ischemic stroke LA is associated with an increased risk of death or dependency, recurrent stroke, intracerebral hemorrhage under anticoagulation, myocardial infarction, and poststroke dementia. There is increasing evidence from neuroimaging studies to support the concept that some cases of LA are caused by white matter infarcts, which may be particularly frequent in patients with widespread small vessel disease. Th e relatively similar distribution of LA regardless of the distribution of vascular pathology suggests a conserved vulnerability to white matter injury across various vascular diseases, possibly related to the resting patterns of blood fl ow (Mijajlovic et al., 2011).

LA is frequently observed in patients with acute stroke, ischemic as well as hemorrhagic. Previous studies indicated that LA was strongly associated with lacunar strokes rather than non-lacunar, territorial strokes. Stroke and LA are likely two related diseases. In many aspects, LA is an ischemic disease, as is ischemic stroke. Also, intracerebral hemorrhage (ICH) and LA share a common cause, that of arterial hypertension. If LA shares with stroke (ischemic and hemorrhagic) common mechanisms, and the appearance of LA on imaging predicts stroke, then, according to the current terminology, LA can be regarded as an intermediate surrogate of stroke. (Jimenez-Conde et al., 2010, Pu et al., 2009, Lee et al., 2008, Inzitari, 2003, as cited by Mijajlovic et al., 2011)

Putaala et al. hypothesized that risk factors, neuroimaging characteristics, and associations with the overt clinical stroke may be diff erent in young patients with ischemic stroke with or without silent brain infarcts (SBIs) and LA. Of the 669 patients included, $86(13 \%)$ had SBIs, $50(7 \%)$ had LA, $17(3 \%)$ had both, and 550 served as controls. Most SBIs were located in basal ganglia $(39 \%)$ or subcortical regions $(21 \%)$, but cerebellar SBIs also were rather frequent $(15 \%)$. LA was mainly mild to moderate. Silent cardioembolism may in part explain the frequency of cerebellar SBIs in younger patients. As observed, younger stroke patients tend to have more frequently overt posterior territory ischemia and cerebellar infarcts. Th is observation, jointly with the frequency of cerebellar SBIs, might reflect the same, yet unclear, pathophysiologic mechanisms. Independent risk factors for SBIs in younger adults were type 1 diabetes, obesity, smoking, and increasing age. Risk factors for LA were type 1 diabetes, obesity, female sex, and increasing age. Small-vessel disease was the predominant cause of stroke in both those with SBIs (31\%) and LA (44\%) (Putaala et al., 2009, as cited by Mijajlovic et al., 2011).

Prospective observations further corroborated the relationship between LA and stroke and the distinct role of lacunar infarcts. The presence of LA on CT scan predicted subsequent stroke in patients with first-ever lacunar stroke, in those with lacunar or cortical infarction, 
in elderly patients with gait problems and LA on CT scan, and in patients with lacunar stroke or trivial neurological symptoms. Recurrent stroke was predominantly of the lacunar type. When studies took into account the severity of LA the risk of recurrence proved to be proportional to the extent of LA (Miyao et al., 1992, van Zagten et al., 1996, Inzitari et al., 1995, Yamauchi et al., 2002, as cited by Inzitari, 2003).
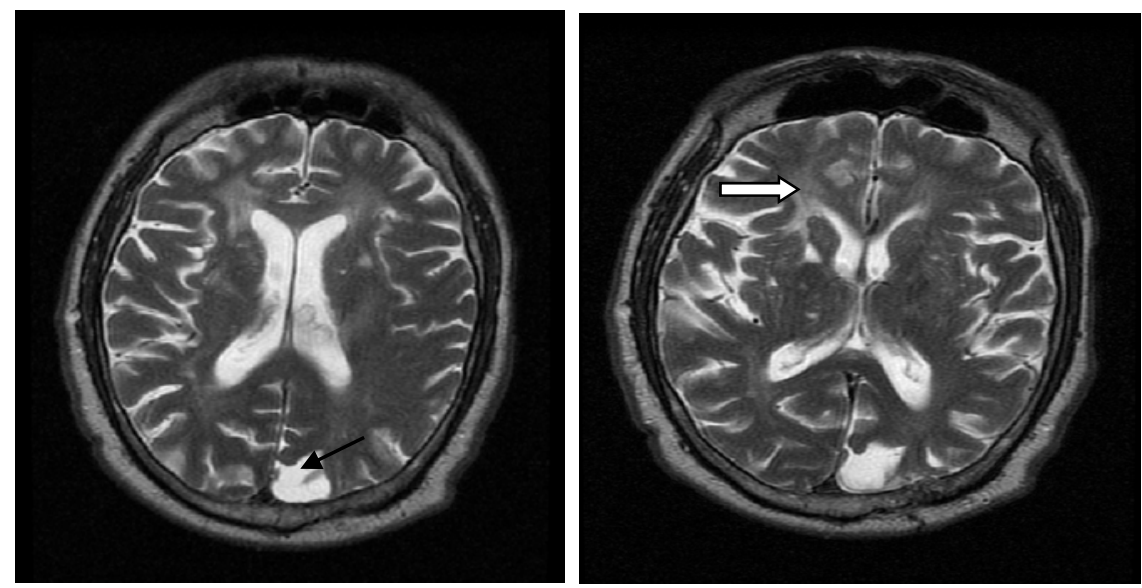

Fig. 3. Cerebral MRI (axial T2 weighted sequences) of a 70 years old male, known with arterial hypertension, diabetes mellitus, and a left posterior cerebral arterial ischemic stroke 3 years ago, admitted for right hemiparesis and mixed aphasia; an old stroke in the left posterior cerebral artery territory (black arrow) and leukoaraiosis (white arrow) can be seen.

In 2008 we attempted to assess the correlation between leukoaraiosis and associated vascular pathologies in a group of 50 hospitalized patients whose cerebral MRI revealed leukoaraiosis. The study revealed that:

- $\quad$ Stage II leukoaraiosis (Brant-Zawadzki scale) was present frequently in patients with acute stroke.

- $\quad$ Stage III leukoaraiosis (Brant-Zawadzki scale) predominated in patients with chronic vascular lesions.

- Stage IV (Brant-Zawadzki scale) in our studied group was found in those with Binswanger disease.

- Severity of leukoaraiosis increased with age.

- Severity of neurologic symptoms was in direct proportion with severity of leukoaraiosis.

- Leukoaraiosis associated cognitive decline and brain atrophy in $66 \%$ of cases.

- Presence of leukoaraiosis was associated with increased risk of stroke recurrence (almost half of the cases) (Hâncu et al., 2009).

\subsection{Large arteries stroke}

In most of the studies, cortical territorial infarct was largely less probable than lacunar or hemorrhagic stroke as recurrent stroke. The main causes of cortical infarcts are large-artery disease and cardioembolism. 


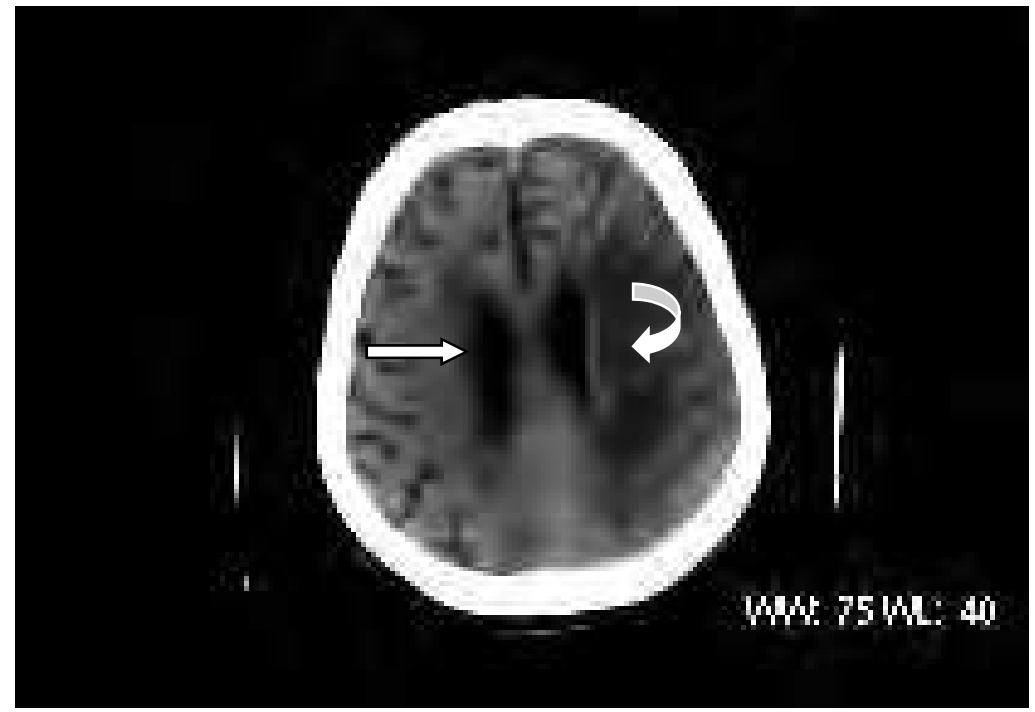

Fig. 4. Cerebral CT scan showing leukoaraiosis (white arrow) in a pacient with an old ischemic stroke in the left middle cerebral artery territory (white curved arrow).

In regard to the first mechanism, while cross-sectional data from the patients randomized in the North American Symptomatic Carotid Endarterectomy Trial (NASCET) showed an inverse relationship between the degree of carotid stenosis and presence of LA, a few population studies have consistently reported an association between intima-media thickness or the presence of carotid plaques and white matter hyperintensities on MRI, even after adjustment for other vascular risk factors. Atrial fibrillation, a major cause of cardioembolic stroke, was found to be negatively associated with LA in 1 study and positively associated in 2 other studies. These conflicting results are likely justified by the variable impact of age and other age-related vascular risk factors as confounders for these associations (Brun \& Englund, 1986, Pico et al., 2002, Bots et al., 1993, Manolio et al., 1999, Henon et al, 1996, Raiha et al., 1993, as cited by Inzitari 2003).

In a Korean study, a significant association between leukoaraiosis and the stroke subtypeswas found. The large-artery-disease group had a higher prevalence of leukoaraiosis than did the other groups (55.4\% in the large-artery-disease group, 30.3\% in the lacunar group and $14.3 \%$ in the cardioembolic group, $\mathrm{P}=0.016$ by chi-square test). On the multivariate linear regression analysis, age, the presence of hypertension, previous stroke and stroke subtype were independently associated with the presence of leukoaraiosis. In the sub analysis of the large-artery-disease group, the leukoaraiosis had a tendency to be more prevalent in the mixed and intracranial stenosis group than did the extracranial stenosis group $(45.5 \%$ in the mixed group, $40.3 \%$ in the intracranial group and $26.9 \%$ in the extracranial group, $\mathrm{P}=0.08$ by chi-square test). The association of leukoaraiosis with largeartery disease in this study might be due to the relatively high prevalence of intracranial occlusive lesions in Korean stroke patients compared to other ethnic groups (Lee et al., 2008). 

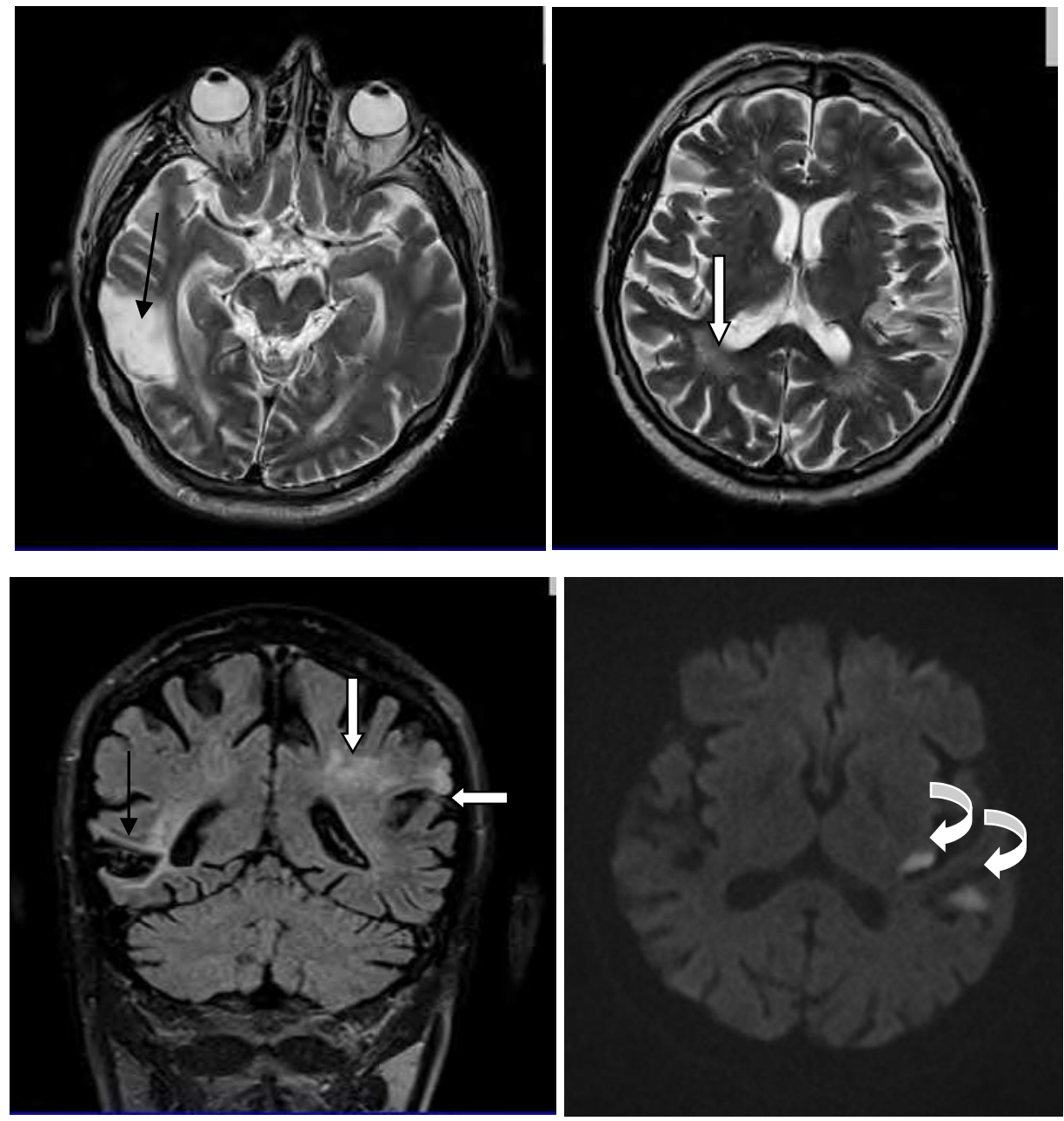

Fig. 5. Cerebral MRI (above: axial T2 weighted sequences, left-below: coronal FLAIR sequence, right-below: diffusion-weighted sequence) showing an old right temporal lobe ischemic stroke (black arrow), acute left temporo-frontal ischemic stroke (white horizontal arrow), leukoaraiosis (white vertical arrow) and diffusion - hypersignal spots in left temporal lobe (curved white arrows).

\subsection{Lacunar stroke}

Although leukoaraiosis and lacunar infarcts are often found together, in individual patients one type of imaging appearance may predominate, leading to the notion of subtypes of diffuse small vessel disease; either what has been labelled as ischaemic leukoaraiosis (defined by the combination of leukoaraiosis with a history of a clinical lacunar syndrome), or isolated lacunar infarction (in which a similar clinical presentation is accompanied by multiple lacunar lesions but no leukoaraiosis on imaging). These two imaging types have 
recently been shown to differ in their risk factor profile; age and hypertension are most strongly associated with ischaemic leukoaraiosis while hypercholesterolaemia, diabetes mellitus and myocardial infarction are more associated with isolated lacunar infarction. These findings suggest some differences in pathogenesis, with leukoaraiosis, perhaps reflecting a non-atheromatous pathology of smaller calibre vessels than those implicated in lacunar infarcts. A better appreciation of distinct subtypes may explain conflicting results about the association of certain novel risk factors with stroke; for example, although homocysteine is a risk factor for both types of manifestation, it has a much stronger association with ischaemic leukoaraiosis than with isolated lacunar infarction (Khan et al., 2007, Hassan at al., 2004, as cited by O' Sullivan, 2008).

White matter hyperintensities on magnetic resonance imaging can be used as surrogate markers of small vessel disease (Wallin \& Fladby, 2010).

Rost et al. measured WMH volume (WMHV) in cohorts of prospectively ascertained patients with acute ischemic stroke (AIS) $(\mathrm{n}=891)$ and ICH $(\mathrm{n}=122)$. Patients with larger WMHV were more likely to have lacunar stroke compared with cardioembolic, large artery, or other stroke subtypes $(p<0.03)$. In a separate analysis, greater WMHV was seen in ICH compared with lacunar stroke and in ICH compared with all ischemic stroke subtypes combined $(p<0.007)$. These data support the model that increasing WMHV is a marker of more severe cerebral small-vessel disease (Rost et al., 2010).
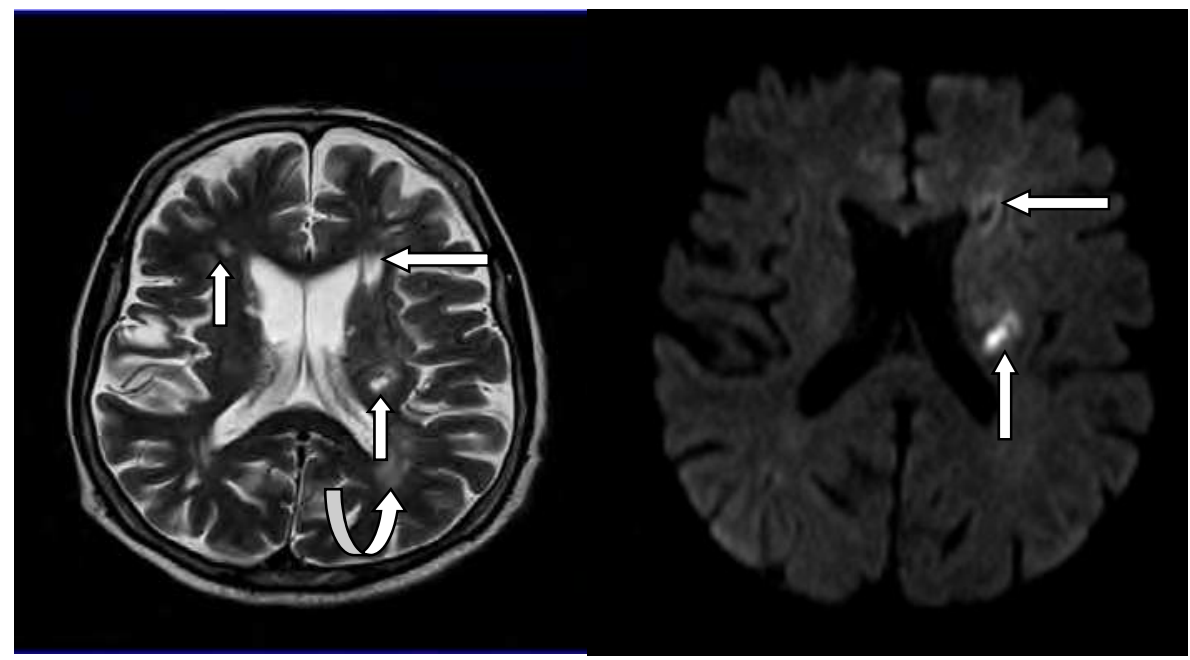

Fig. 6. Cerebral MR scan of a 60 years old female (left: T2 weighted sequence; right: diffusion weighted sequence) showing an old microbleeding (horizontal arrow), lacunar ischemic strokes (vertical arrow) and leukoaraiosis (curved arrow).

\subsection{Hemorrhagic stroke}

2 cross-sectional studies of hospitalized stroke patients reported in 1989 and 1990, for the first time, the association between LA and ICH. The latter of the 2 studies also examined the possible confounders for this association. In this study extensive LA was over twice more 
prevalent among 116 patients with ICH than among 155 control patients without ICH. In an analysis with a multivariate adjustment for other vascular risk factors, the association was almost fully explained by the higher prevalence of arterial hypertension and lacunar infarcts on CT scan (Selekler \& Erzen, 1989, Inzitari et al., 1990, as cited by Inzitari, 2002).

The Stroke Prevention in Reversible Ischemia Trial (SPIRIT), a prospective trial of secondary prevention with anticoagulation and target international normalized ratio (INR) values of 3.0 to 4.5 in patients with cerebral ischemia of presumed arterial origin, revealed the independent role of LA as a risk factor for major bleeding during anticoagulation after cerebral ischemia (Gorter, 1999). This was confirmed by a recent study which investigated radiographic and clinical characteristics of patients with warfarin-related ICH following ischemic stroke. The 26 eligible ICH cases and 56 controls were compared for vascular risk factors, stroke characteristics, and extent of leukoaraiosis (graded in anterior and posterior brain regions on a validated scale of 0 to 4 ). Leukoaraiosis was found to be an independent risk factor for warfarin-related $\mathrm{ICH}$ in survivors of ischemic stroke, including those in the commonly employed range of anticoagulation (Smith et al., 2010).
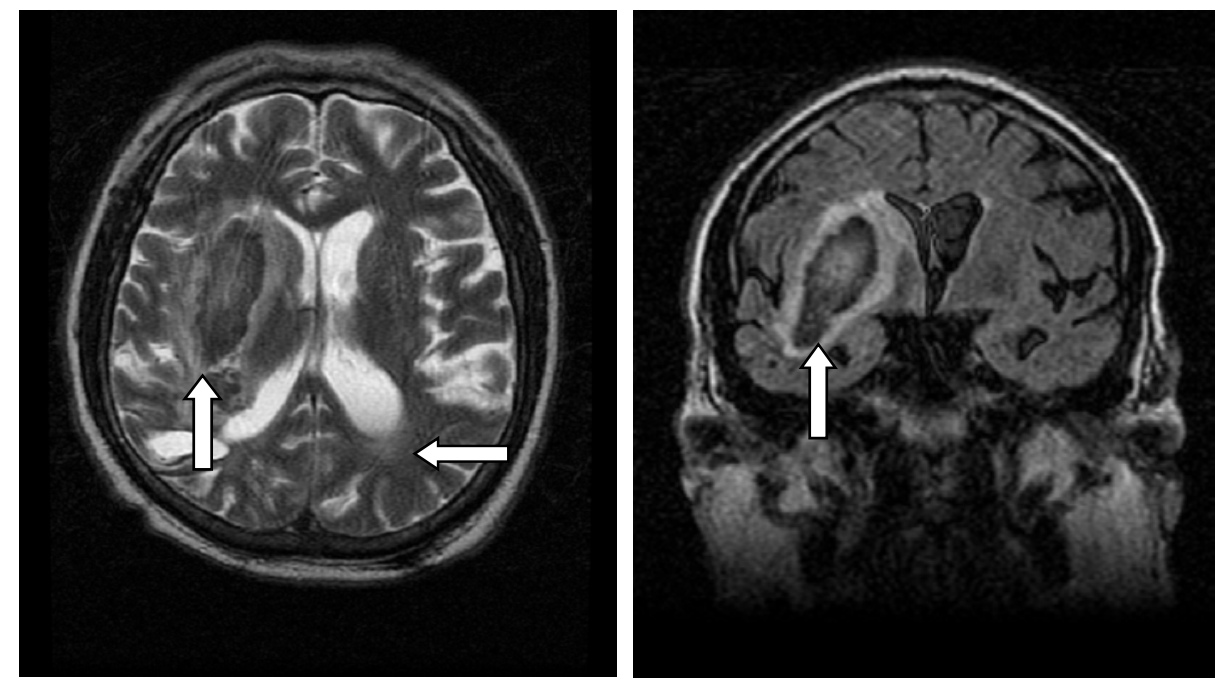

Fig. 7. Cerebral MRI scan (left: axial T2 weighted sequence; right: coronal FLAIR image) showing right temporo-parietal primary intracerebral hematoma (vertical arrow), mixed cerebral atrophy, leukoaraiosis (horizontal arrow), in a 65 years old male, known with untreated arterial hypertension, smoker, admitted for left hemiplegia suddenly occurred.

\subsection{CADASIL}

The distribution of white matter lesions in cerebral autosomal dominant arteriopathy with subcortical infarcts and leukoaraiosis (CADASIL) has been reported to be distinct from those in patients with ischemic leukoaraiosis and Binswanger's disease. In earlier European studies, diagnostic significance of white matter lesions in the temporopolar region (Tp), medial frontopolar region (Fp) and external capsule (EC) was stressed in diagnosing CADASIL (Tomimoto et al., 2005). 
More recently, however, high sensitivity and specificity of $\mathrm{Tp}$ lesions have been demonstrated. Tomimoto et al examined the frequencies of CADASIL-associated lesions in 17 non-demented patients with ischemic leukoaraiosis and 20 patients with Binswanger's disease. The results indicated that $\mathrm{Tp}$ lesions were useful diagnostic marker in diagnosing CADASIL, whereas Fp and EC lesions were non-specifically observed (Tomimoto et al., 2005).

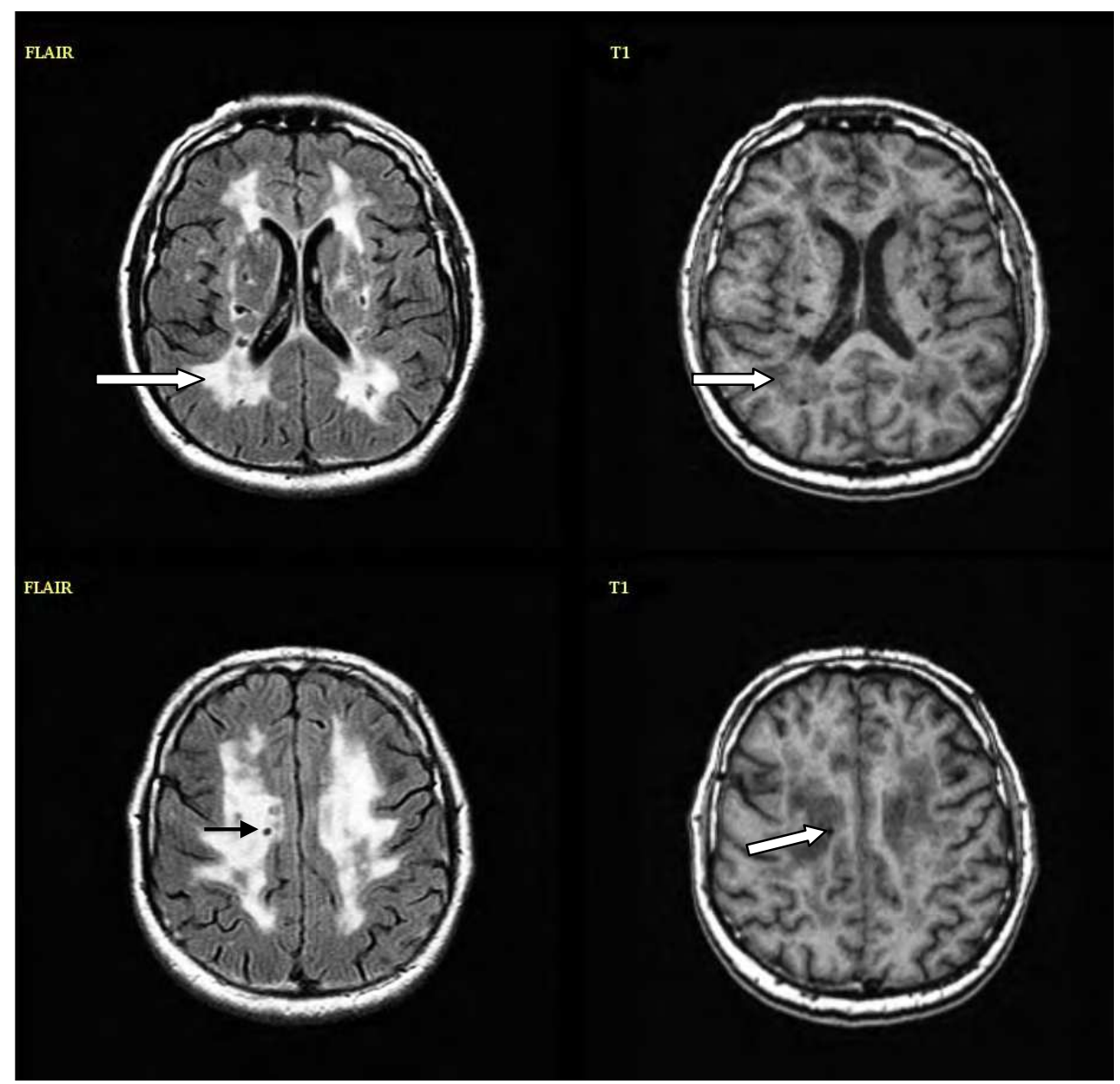

Fig. 8a. MR images from a 24-year old man with CADASIL; left: FLAIR diffuse hypersignal of the periventricular and deep white matter (leukoaraiosis) and small cerebrospinal fluid intensity area (lacunar subcortical infarction); right: T1 hyposignal (leukoaraiosis) and fluid intensity area (lacunar subcortical infarction) in the corresponding regions.

An intersting feature of leukoaraiosis, with possible relevance to the pathogenesis of the lesions, is the relatively conserved anatomic distribution across individuals and different vascular diseases. Exceptions are that in CADASIL, there is relative preferential 
involvement of the external capsule and anterior temporal white matter; and in cerebral amyloid angiopathy, there is relatively more posterior than anterior leukoaraiosis. Even so, in CADASIL and cerebral amyloid angiopathy patients, as well as others, the greatest burden of lesions is in the periventricular white matter around the frontal and occipital horns, with varying amounts of involvement of areas of subcortical white matter. The probability of subcortical white matter involvement varies inversely with the distance from the ventricular margin (Smith, 2010).

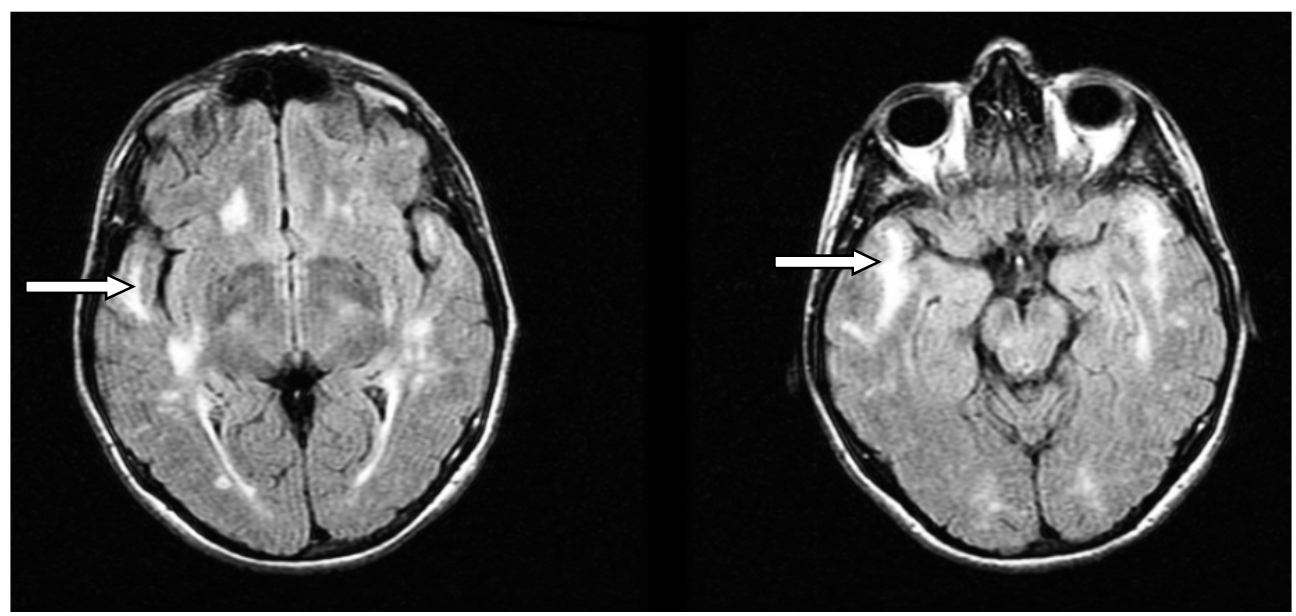

Fig. 8b. Same case: FLAIR hypersignal in the anterior pole of the temporal lobe, characteristic for CADASIL.

\subsection{Amyloid angiopathy}

Cerebral amyloid angiopathy affects the arteries of the leptomeninges and cortex, with little involvement of the penetrating arteries in the white matter and essentially no involvement of the perforating arteries at the base of the brain and brain stem (Smith 2010).

In Alzheimer's disease, and in patients with mild cognitive impairment, some of whom will have prodromal Alzheimer's, white matter changes correlate with serum levels of the Ab $1-40$ peptide, which is the predominant peptide found in vessel deposits in cerebral amyloid angiopathy. But intriguingly, plasma $\mathrm{Ab}$ also correlates with the severity of white matter lesions in the population-based Rotterdam study. Given that cerebral amyloid angiopathy is a rare cause of leukoaraiosis at the population level, this suggests that $\mathrm{Ab}$ has an impact on white matter damage even in patients with arteriosclerotic small vessel disease and no pathological evidence of cerebral amyloid angiopathy. The underlying mechanisms of this association are not yet clear. Conversely, pathological studies in Alzheimer's disease suggest that arteriosclerotic small vessel disease can drive more extensive amyloid deposition and neurofibrillary tangle formation, which is consistent with epidemiological evidence that vascular risk factors are important, and suggests that the interaction may work in both directions (Gurol et al., 2006, van Dijk et al., 2004, Thal et al., 2003, as cited by O' Sullivan, 2008). 
Leukoaraiosis is also increasingly recognised as a feature of sporadic cerebral amyloid angiopathy, without Alzheimer's disease.Amyloid deposits are found in more proximal portions of the small penetrating arteries than arteriosclerotic changes and it is not known whether these deposits alone, without downstream arteriosclerosis, are sufficient to cause leukoaraiosis. Notably, leukoaraiosis is found in some of the genetic amyloid angiopathies (for example, familial British dementia) suggesting that amyloid angiopathy alone is sufficient to cause this imaging appearance (Greenberg et al., 2004, as cited by O' Sullivan, 2008).
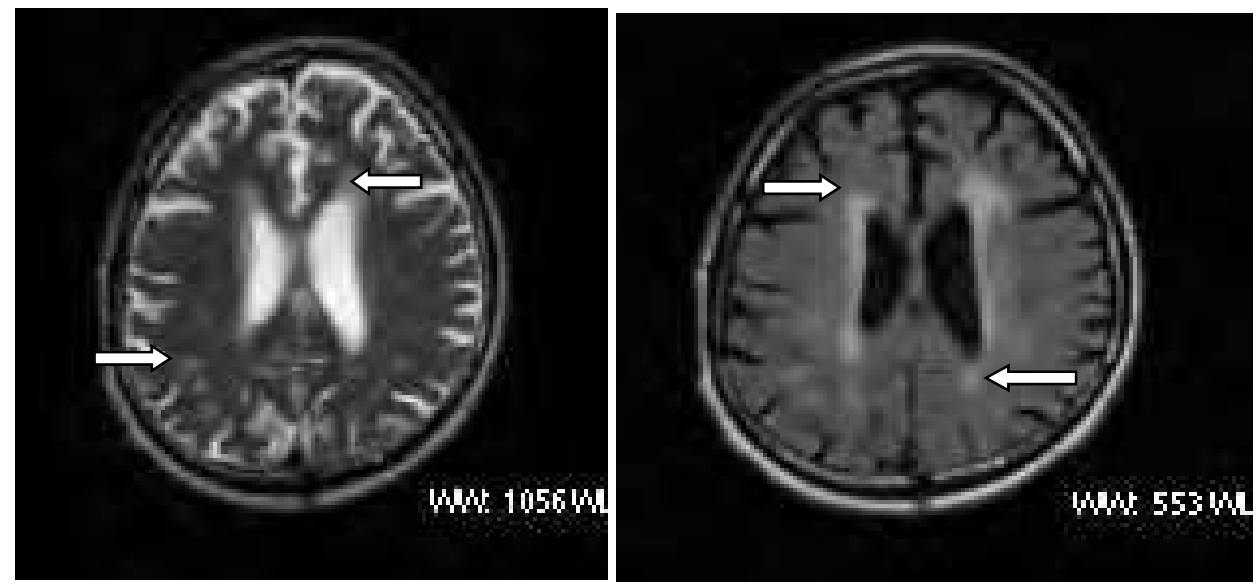

Fig. 9a. MR images from a 71-year-old man with probable cerebral amyloid angiopathy and leukoaraiosis; left: T2 hypersignal at the level of periventricular and deep white matter; right: FLAIR hypersignal at the level of the periventricular white matter.
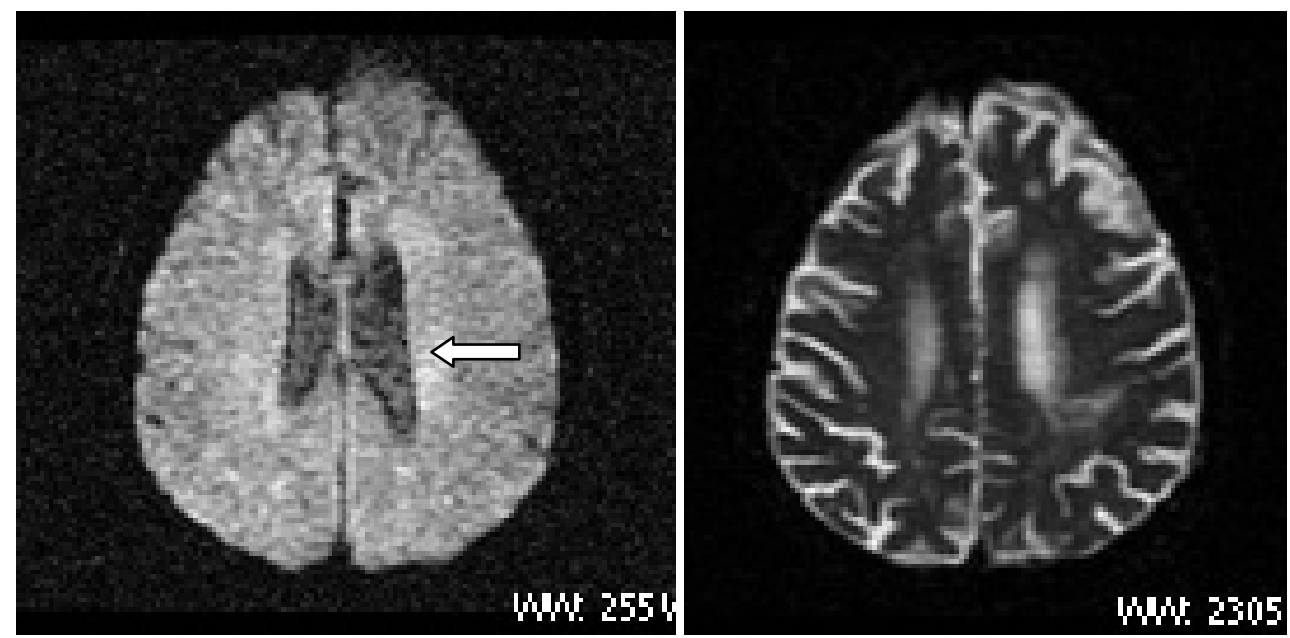

Fig. 9b. Same case: MR diffusion weighted image (DWI) and apparent diffusion coefficient (ADC) mapping; no apparent diffusion coefficient (ADC) restriction can be observed in the affected areas (leukoaraiosis). 


\subsection{Collagen arteritis}

Neurologic manifestations are among the features of many multisystem autoimmune connective tissue disorders with various clinical presentations. Areas of patchy cortical or subcortical abnormality (hyperdensity on CT scan, T2 hyperintensity on MRI) may correspond to small vessel vasculitis or cerebritis (Ramachandran, 2011).
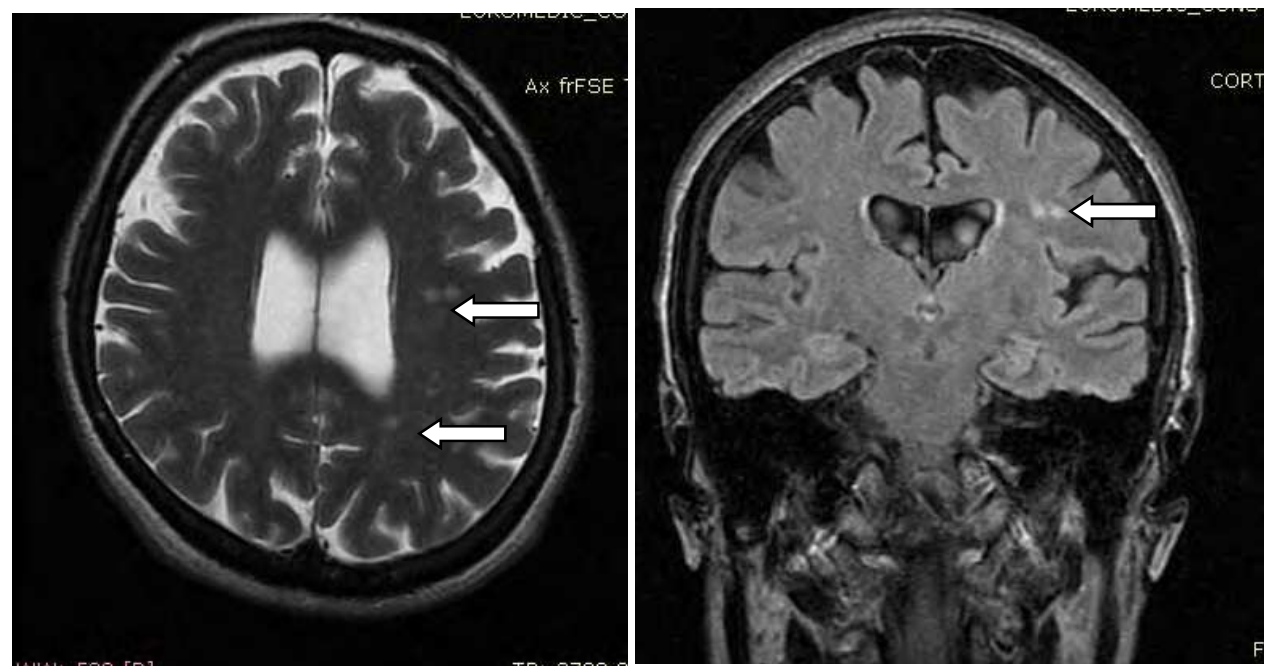

Fig. 10. MR images from a 51-year-old female with rheumathoid arthritis; T2 hypersignal (left) and FLAIR hypersignal (right) spots at the level of deep white matter can be seen.

\section{Conclusions}

Leukoaraiosis is a radiological finding whose pathogenesis or clinical significance is not completely acknowledged. LA predispose to stroke, independent of other stroke risk factors. Typically, leukoaraiosis predict a lacunar ischemic stroke but in patients with LA the risk of cortical infarct is also increased, as is that of vascular death. The presence of extensive LA predisposes to $\mathrm{ICH}$, especially in patients treated with anticoagulants for secondary prevention after an ischemic stroke. Consequently, it is important for practicians to correctly assess LA in all patients presenting with cerebro-vascular pathology.

\section{Acknowledgements}

We are grateful to Dr. Virgil Ionescu and Dr. Mariana Bardaş for the images of this chapter.

\section{References}

Birns J. \& Kalra L. (2008).Pathogenesis of Cerebral Small Vessel Disease, In: Brain HypoxiaIschemia Research Progress, O.M. Roux, (Ed.), 113-130, Nova Science Publisher Inc., ISBN: 978-1-60456-139-5, New York, USA 
Bohnen, N.I.; Bogan C.W. \& Muller M.L.T.M. (2009). Frontal and Periventricular Brain White Matter Lesions and Cortical Deafferentation of Cholinergic and Other Neuromodulatory Axonal Projections. European Neurological Journal, No. 1, pp. 1-7

Brown W.R., MoodyD.M., Challa V.R., Thore C.R. \& Anstrom J.A. (2002). Apoptosis in leukoaraiosis lesions

Candelario-Jalil E., Thompson J., Taheri S., Grossetete M., Adair J.C., Edmonds E., Prestopnik J., Wills J., Rosenberg G.A. (2011). Matrix metalloproteinases are associated with increased blood-brain barrier opening in vascular cognitive impairment. Stroke, Vol. 42, No. 5, (May 2011), pp. 1345-50, ISSN 1524-4628

Chung C.P. \& Hu H.H. (2010).Pathogenesis of leukoaraiosis: Role of jugular venous reflux. Medical Hypotheses, Vol. 75, No. 1, (July 2010), pp. 85-90

Debette, S., \& Markus, H. (2010). The clinical importance of white matter hyperintensities on brain magnetic resonance imaging: systematic review and meta-analysis. BMJ, No. 341 (July 2010), c3666.

Smith E.E. (2010). Leukoaraiosis and Stroke. Stroke, Vol. 75, No. 1, (July 2010), pp. 85-90

Fazekas F., Kleinert R., Offenbacher H., Schmidt R., Kleinert G., Payer F., Radner H. \& Lechner H. (1993). Pathologic correlates of incidental MRI white matter signal hyperintensities. Neurology, No.43, (September 1993), pp. 1683-9

Fernandez-Cadenas I, Mendioroz M, Domingues-Montanari S, Del Rio-Espinola A, Delgado P, Ruiz A, Hernandez-Guillamon M, Giralt D, Chacon P, Navarro-Sobrino M, Ribo M, Molina CA, Alvarez-Sabin J, Rosell A, Montaner J. (2010). Leukoaraiosis is associated with genes regulating blood-brain barrier homeostasis in ischaemic stroke patients. European Journal of Neurology, No. 18, (December 2010), pp. 826-35

Fujita S., Kawaguchi T., Uehara T. \& Fukushima K. (2005). Progress of leukoaraiosis is inhibited by correction of platelet hyper-aggregability. International psychogeriatrics, Vol 17, No. 4, (December 2005), pp. 689-98, ISSN: 1041-6102

Gomes J.A. \& Caplan L.R. White matter abnormalities in patients with cerebrovascular disease, In: MR imaging in white matter diseases of the brain and spinal cord, $M$. Filippi, (Ed.), 364-71, Springer, ISBN 3-540-40230-6, Heidelberg , Germany

Gorter J.W. (1999). For the Stroke Prevention in Reversible Ischemia Trial (SPIRIT) and European Atrial Fibrillation Trial (EAFT) Study Groups. Major bleeding during anticoagulation after cerebral ischemia: patterns and risk factors. Neurology, Vol. 53, (October 1999), pp. 1319-1327

Hachinski V.C., Potter, P. \& Merskey, H. (1987). Leuko-araiosis. Archives of Neurology, No. 44, pp. 21-23

Hâncu A., Damian I., Popescu S., Davidescu B.H., Zguma D., Herțea C. \& Kaivanifard M. (2009). Impact of imaging development upon leukoaraiosis detection in a group of vascular patients. Romanian Journal of Neurology, Vol. VIII, No. 3, (November 2009), pp. 109-114, ISSN 1843-8148

Inzitari D. Leukoaraiosis An Independent Risk Factor for Stroke? (2003). Stroke, Vol. 34, (June 2003), pp. 2067-2071 Journal of the Neurological Sciences, Vol. 203-204, (November 2002), pp. 169-171

Kurkinen M. (September 2009). Silent brain infarcts and leukoaraiosis in young patients with first-ever ischemic stroke. Available from https://helda.helsinki.fi

LeeS.J., Kim J.S., Lee K.S., An J.Y., Kim W., Kim Y.I., Kim B.S. \& Jung S.L. (2008). The leukoaraiosis is more prevalent in the large artery atherosclerosis stroke subtype 
among Korean patients with ischemic stroke. BMC Neurology, Vol. 8, No. 1, (August 2008), pp. 31-33.

Lotz P.R., Ballinger W.E. Jr \& Quisling R.G. (1986). Subcortical arteriosclerotic encephalopathy: CT spectrum and pathologic correlation. American Journal of Roentgenology, No. 147, (December 1986), pp. 1209-14

Martí-Fàbregas J., Valencia C., Pujol J., García-Sánchez C. \& Martí-Vilalta J.L. (2002). Fibrinogen and the amount of leukoaraiosis in patients with symptomatic smallvessel disease. European Neurology, Vol. 48, No. 4, (January 2002), pp. 185-90.

Mijajlovic M.D., Pavlovic A.M., Mircovic M.M. \& Sternic N. (2011). Connection Between Leukoaraiosis And Ischemic Stroke. Current Topics in Neurology and Psychiatry Related Disciplines, Vol. 19, No. 1, (March 2011), pp. 41-47

Murata T, Handa H, Mori K, Nakano Y. (1981). The significance of periventricular lucency on computed tomography: experimental study with canine hydrocephalus. Neuroradiology, No. 20, (January 1981), pp. 221-227

O' Sullivan M, Summers P.E., Jones D.K., Jarosz J.M., Williams S.C.R. \& Markus H.S. (2001). Normal appearing white matter in ischemic leukoaraiosis: a diffusion tensor MRI study. Neurology, No. 57, (December 2001), pp. 2307-10

O' Sullivan, M. (2008). Leukoaraiosis. Practical Neurology, No. 8, pp. 26-38

O'Sullivan M., Lythgoe D. J., Pereira ., Summers P. E., Jarosz J. M., Williams S. C.R. \& Markus H.S. (2002). Patterns of cerebral blood flow reduction in patients with ischemic leukoaraiosis. Neurology, No. 59, (August 2002), pp. 321-6

Ramachandran T.S. (29 june 2011). Neurologic Manifestations of Systemic Lupus. Available from http://emedicine.medscape.com/article/1146456-overview

Ropele S., Enzinger C., Söllinger M., Langkammer C., Wallner-Blazek M., Schmidt R. \& Fazekas F. (2010). Impact of Sex and Vascular Risk Factors on Brain Tissue Changes with Aging: Magnetization Transfer Imaging Results of the Austrian Stroke Prevention Study. American Journal of Neuroradiology, No. 31, (August 2010), pp. 1297-1301

Rost N.S., Rahman R.M., Biffi A., Smith E.E., Kanakis A., Fitzpatrick K., Lima F., Worrall B.B., Meschia J.F., Brown R.D. Jr, Brott T.G., Sorensen A.G., Greenberg S.M., Furie K.L. \& Rosand J. (2010). White matter hyperintensity volume is increased in small vessel stroke subtypes. Neurology, Vol. 75, No. 19, (November 2010), pp. 1670-1677

Saba L., Pascalis L., Sanfilippo R., Anzidei M., Bura R., Montisci R. \& Mallarini G. (2011). Carotid Artery Wall Thickness and Leukoaraiosis: Preliminary Results Using Multidetector Row CT Angiography. American Journal of Neuroradiology, No. 32, (May 2011), pp. 955-61

Saba L., Sanfilippo R., Pascalis L., Montisci R. \& Mallarini G. (2009). Carotid Artery Abnormalities and Leukoaraiosis in Elderly Patients: Evaluation with MDCT. American Journal of Roentgenology, No. 192, (February 2009), pp. W63-W70

Schneider R, Ringelstein EB, Zeumer H, Kiesewetter H, Jung F. (1987). The role of plasma hyperviscosity in subcortical arteriosclerotic encephalopathy (Binswanger's disease). Journal of Neurology, No. 234, (December 1987), pp. 67-73

Smith E.E., Rosand J., Knudsen K.A., Hylek E.M. \& Greenberg S.M. (2002). Leukoaraiosis is associated with warfarin-related hemorrhage following ischemic stroke. Neurology, Vol.59, No. 2, (July 2002),pp. 193-197 
Tomimoto H., Ohtani R., Wakita H., Lin J.X., Miki Y. \& Mizuno T. (2005). Distribution of ischemic leukoaraiosis in MRI: a difference from white matter lesions in CADASIL. Brain and nerve, Vol. 57, No. 2, (February 2005), pp. 125-130

Wallin A. \& Fladby T. (2010). Do white matter hyperintensities on MRI matter clinically?BMJ, Vol. 341, (July 2010), pp. C 3400

Xu H., Stamova B., Jickling G., Tian Y., Zhan X., Ander B.P., Liu D., Turner R., Rosand J., Goldstein L.B., Furie K.L., Verro P., Johnston S.C., Sharp F.R. \& DeCarli C.S. (2010). Distinctive rna expression profiles in blood associated with white matter hyperintensities in brain. Stroke, No. 41, (October 2010), pp. 2744-49 


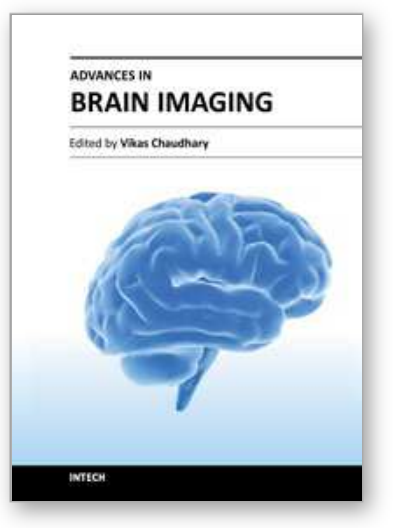

\author{
Advances in Brain Imaging \\ Edited by Dr. Vikas Chaudhary
}

ISBN 978-953-307-955-4

Hard cover, 264 pages

Publisher InTech

Published online 01, February, 2012

Published in print edition February, 2012

Remarkable advances in medical diagnostic imaging have been made during the past few decades. The development of new imaging techniques and continuous improvements in the display of digital images have opened new horizons in the study of brain anatomy and pathology. The field of brain imaging has now become a fast-moving, demanding and exciting multidisciplinary activity. I hope that this textbook will be useful to students and clinicians in the field of neuroscience, in understanding the fundamentals of advances in brain imaging.

\title{
How to reference
}

In order to correctly reference this scholarly work, feel free to copy and paste the following:

Anca Hâncu, Irene Răşanu and Gabriela Butoi (2012). White Matter Changes in Cerebrovascular Disease: Leukoaraiosis, Advances in Brain Imaging, Dr. Vikas Chaudhary (Ed.), ISBN: 978-953-307-955-4, InTech, Available from: http://www.intechopen.com/books/advances-in-brain-imaging/white-matter-changes-incerebrovascular-disease-leukoaraiosis

\section{INTECH}

open science | open minds

\section{InTech Europe}

University Campus STeP Ri

Slavka Krautzeka 83/A

51000 Rijeka, Croatia

Phone: +385 (51) 770447

Fax: +385 (51) 686166

www.intechopen.com

\section{InTech China}

Unit 405, Office Block, Hotel Equatorial Shanghai

No.65, Yan An Road (West), Shanghai, 200040, China

中国上海市延安西路65号上海国际贵都大饭店办公楼 405 单元

Phone: +86-21-62489820

Fax: +86-21-62489821 
(C) 2012 The Author(s). Licensee IntechOpen. This is an open access article distributed under the terms of the Creative Commons Attribution 3.0 License, which permits unrestricted use, distribution, and reproduction in any medium, provided the original work is properly cited. 